CLNS-00/1710

\title{
STRING WEBS AND THE DECAY OF SUPERSYMMETRIC PARTICLES
}

\author{
PHILIP C. ARGYRES and K. NARAYAN \\ Newman Laboratory, Cornell University \\ Ithaca, NY 14853, USA
}

\begin{abstract}
The spectrum of stable electrically and magnetically charged supersymmetric particles can change discontinuously due to the decay of these particles as the vacuum on the Coulomb branch is varied. We show that this decay process is well described by semiclassical field configurations purely in terms of the low energy effective action on the Coulomb branch even when it occurs at strong coupling. The resulting picture of the stable supersymmetric spectrum is a generalization of the "string web" picture of these states found in string constructions of certain theories.
\end{abstract}

Four dimensional gauge theories with at least eight supersymmetries have a Coulomb branch of inequivalent vacua in which the low energy theory has unbroken $U(1)$ gauge factors. These theories also have a spectrum of massive charged particles with various electric and magnetic charges under the $U(1)$ 's, lying in supersymmetry multiplets. The masses of those in short multiplets - the BPS states - are related to their charges by the supersymmetry algebra.t The spectrum of possible BPS masses can then be determined using supersymmetry selections rules 3 This, however, leaves open the question of the existence and multiplicity of these states. Furthermore, even if such a state exists in some region of the Coulomb branch, it may be unstable to decay at curves of marginal stability (CMS) on the Coulomb branch 3 We propose 4 a solution to the question of the multiplicity of BPS states for $N=2$ supersymmetric theories in four dimensions just in terms of the low energy effective $U(1)^{n}$ action on the Coulomb branch.

The form of the answer we get coincides with the "string web" picture of BPS states developed in the context of the D3-brane construction of $N=4 S U(n)$ superYang-Mills theory and the F-theory solution to $N=2 S U(2)$ gauge theory with fundamental matter 5 . Moreover, our solution generalizes these constructions to arbitrary field theory data (gauge groups, matter representations, couplings and masses). The resulting picture is quite simple: BPS states are represented by string webs on the Coulomb branch of the theory with one end at the point corresponding to the vacuum in question (the analog of the 3-brane probe in the F-theory picture) and the other ends lying on the complex codimension 1 singularities on the Coulomb branch (the analogs of the $(p, q)$ 7-branes of the F-theory picture). The strands of the string web lie along geodesics in the Coulomb branch metric. Each strand carries electric and magnetic charges under the $U(1)^{n}$ low energy gauge group: the total charge flowing into the vacuum point determines the total charge of the BPS state, while only multiples of the charges determined by the $S p(2 n, Z)$ monodromies 
around the codimension 1 singularities are allowed to flow into those ends of the web.

Perhaps the most surprising thing about our solution is that it describes the stability of the monopole and dyon BPS spectrum wholly in terms of the $U(1)^{n}$ low energy effective action. This is possible because the distance $\Delta X$ from a given vacuum on the Coulomb branch to a CMS acts as a new low energy scale which can be made arbitrarily small compared to the strong coupling scale $\Lambda$ of the nonAbelian gauge theory as we approach the CMS. In particular, we show that as we approach the CMS the classical low energy field configuration describing a state which decays across the CMS will develop two or more widely separated charge centers (which appear as singularities in the low energy solution); the distance between these centers varies inversely with $\Delta X$. In this limit the details of the microscopic physics become irrelevant for the decay of a BPS state across a CMS, which is described by a low energy field configuration with charge centers becoming infinitely separated.

The classical BPS field configuration of the scalar fields in the low energy $U(1)^{n}$ theory will have one or more singularities or sources where scalar field gradients and $U(1)$ field strengths diverge. Near these points the low energy description breaks down and should be cut off by boundary conditions reflecting the matching onto the microscopic physics of the non-Abelian gauge theory. We show that these boundary conditions are determined by the BPS condition.

Several previous discussions 6 目 of the semi-classical description of BPS states in supersymmetric theories were the starting point for this work.

\section{BPS states near CMS}

We restrict our attention to a single charge sector of the low energy $U(1)^{n}$ theory on the Coulomb branch. The question is whether at a given vacuum there is or is not a one-particle BPS state in that charge sector. The mass of a BPS state is $M=|Z|$ where the central charge $Z$ is the sum of terms proportional to the charges. This mass is the minimum mass of any state (BPS or not, single particle or not) in this charge sector. A single BPS particle $M$ in this charge sector is stable or at worst marginally stable against decay into two (or more) constituent particles, since by charge conservation $Z=Z_{1}+Z_{2}$, so by the triangle inequality $M \leq M_{1}+M_{2}$. The CMS are submanifolds of the Coulomb branch where this inequality is saturated. As one adiabatically changes the order parameter on the Coulomb branch from a vacuum on one side of a CMS to a vacuum on the other, the one particle state $M$ becomes more and more nearly degenerate with the two particle state $M_{1}+M_{2}$. Suppose $M$ does decay across the CMS, so only the two particle state $M_{1}+M_{2}$ remains in the spectrum once we have crossed the CMS. Since the transition takes place right at threshold, $M$ will decay into the two particle state with zero relative momentum.

Now, it follows from the triangle inequality that the two particle state $M_{1}+M_{2}$ is not BPS, even for zero relative momentum, except precisely at the CMS. Thus 
there will generically be no BPS force cancelation between particles $M_{1}$ and $M_{2}$, so the zero relative momentum two particle state is classically approximated by two spatially infinitely separated one-particle states (to have a static configuration). By locality and the adiabatic theorem, the transition across the CMS of a one particle state to a widely separated, zero momentum, two particle state should go by way of field configurations with large spatial overlap, implying that a state decaying just where its mass reaches the two-particle threshold of its decay products should have a diverging spatial extent as it approaches the transition. This argument gives the basic physics underlying our approach to decays across CMS: if a BPS state does decay across a CMS, that decay will be visible semiclassically in the low energy effective action even if it takes place at strong coupling from a microscopic point of view. The resulting picture is quite intuitive: a BPS state decaying across a CMS does so by becoming an ever-larger, more loosely bound state of its eventual decay products. Once the CMS is crossed, the bound state ceases to exist, and so, in particular, there will be no static BPS solutions to the low energy equations of motion and boundary conditions in this region of the Coulomb branch.

\section{A $U(1)$ toy example}

We illustrate these properties in a simple model which captures the main physics of the CMS. Consider the $U(1)$ effective action with two real scalars $X, Y$ :

$$
S=-\int d^{4} x\left(\frac{1}{4} F_{\mu \nu} F^{\mu \nu}+\frac{1}{2} \partial_{\mu} X \partial^{\mu} X+\frac{1}{2} \partial_{\mu} Y \partial^{\mu} Y\right)
$$

This theory by itself is too simple to be interesting, but all we need to add to it to capture the essential physics of the decay of BPS states across CMS are the presence of singularities in the vacuum manifold. In this case the vacuum manifold (Coulomb branch) is the $X-Y$ plane. For concreteness we posit that there are two singularities at points on the Coulomb branch with coordinates $(X, Y)=(L, 0)$ where a particle with electric charge $\left(Q_{E}, Q_{B}\right)=(1,0)$ becomes massless, and $(X, Y)=(0, L)$ where a particle with magnetic charge $\left(Q_{E}, Q_{B}\right)=(0,1)$ becomes massless.

The low energy effective action (1) includes only the first terms in a derivative expansion with higher terms suppressed by powers of a cutoff energy scale $\Lambda$, or distance scale $r_{\Lambda} \equiv \frac{1}{\Lambda}$. Our strategy will be to use the low energy $U(1)$ solution away from the charge cores $\left(r>r_{\Lambda}\right)$ and impose appropriate boundary conditions in the vicinity of the cores $\left(r \sim r_{\Lambda}\right)$. The qualitative features of these boundary conditions are easy to deduce. The basic boundary condition on the electric and magnetic fields follow from Gauss' law, $\oint \mathbf{E} \cdot d \mathbf{a}=4 \pi Q_{E}$, and $\oint \mathbf{B} \cdot d \mathbf{a}=4 \pi Q_{B}$, where the integrals are over spheres enclosing the charge cores.

The $X, Y$ scalar fields tend to approach their values at Coulomb branch singularities near the charge cores. Indeed, such field configurations are energetically favored because they approach those of the charged BPS states which become massless at the singularities $(X, Y)=(0, L)$ or $(L, 0)$. Thus the scalar fields satisfy the 
approximate Dirichlet boundary conditions

$$
(X, Y) \simeq(0, L) \text { or }(L, 0) \text { within } B_{\Lambda}^{3}
$$

where $B_{\Lambda}^{3}$ is a ball of approximate radius $r_{\Lambda}$ around each charge core and $(X, Y) \simeq$ $(L, 0)$ means that $(X, Y)$ pass within a distance $\Lambda$ of $(L, 0)$ on the Coulomb branch. This "fuzzy ball" boundary condition is all we can physically demand of the low energy solution since it is not accurate on spatial resolutions less than $r_{\Lambda}$ nor for field value resolutions less than $\Lambda$. In the limit as the vacuum approaches a CMS, as we have described qualitatively in the last section and will see explicitly below, the size of the field configuration grows, and so the relative size of the cutoff region $r_{\Lambda}$ to the field configuration shrinks. In this limit the fuzzy ball Dirichlet boundary conditions become precise.

To simplify the algebra and make the basic points clear we choose the vacuum to be symmetrically placed at the point $\left(X_{0}, X_{0}\right)$ on the Coulomb branch and choose the state to have electric and magnetic charges $\left(Q_{E}, Q_{B}\right)=(1,1)$. There will then be separate electric and magnetic charge cores. The usual BPS equations for a static field configuration following from (11) are then simply $\nabla^{2} X=\nabla^{2} Y=0$ while the electric and magnetic fields are determined in terms of $X$ and $Y$ by $\mathbf{E}=\nabla X$, and $\mathbf{B}=\nabla Y$. The Gauss constraint implies a solution of the approximate form

$$
X-X_{0}=\frac{1}{\left|\mathbf{x}-\mathbf{x}_{E}\right|}, \quad Y-X_{0}=\frac{1}{\left|\mathbf{x}-\mathbf{x}_{B}\right|}
$$

so that the electric and magnetic charge cores are located at $\mathbf{x}_{E}$ and $\mathbf{x}_{B}$ respectively. We want to check whether there are values of $\mathbf{x}_{E}$ and $\mathbf{x}_{B}$ for which this solution satisfies the fuzzy ball Dirichlet boundary condition (2), which demands that the $(X, Y)$ values taken by a solution enters a small ball around $(L, 0)$ as $\mathbf{x} \rightarrow \mathbf{x}_{E}$ (and a small ball around $(0, L)$ as $\left.\mathbf{x} \rightarrow \mathbf{x}_{B}\right)$.

Now for $\left|\mathbf{x}-\mathbf{x}_{E}\right| \simeq\left(L-X_{0}\right)^{-1}$ it follows that $X \simeq L$ as desired near the electric singularity. But this then implies that $Y \simeq X_{0}+r_{E B}^{-1}$ where $r_{E B} \equiv\left|\mathbf{x}_{E}-\mathbf{x}_{B}\right|$ is the spatial distance between the charge centers. The fuzzy Dirichlet boundary condition $Y \simeq 0$ then implies that the separation of the charge centers must be fixed to be

$$
r_{E B}=-\frac{1}{X_{0}} .
$$

There are two striking things about this conclusion. First, there is only a solution for $X_{0}<0$. This inequality is equivalent to saying that there is only a solution on one side of the CMS for our dyonic $\left(Q_{E}, Q_{B}\right)=(1,1)$ state (as can be checked from the explicit BPS mass formula). Second, as $X_{0} \rightarrow 0^{-}$(i.e. as we approach the CMS) the spatial separation of the electric and magnetic charge centers goes to infinity. This confirms the qualitative description of the decay of a supersymmetric state near the CMS given above.

As we describe in detail elsewherel, in the limit as the vacuum approaches the CMS, the projection on the Coulomb branch of the above low energy solution more 
and more accurately approximates a string web, i.e. it degenerates to a collection of segments of geodesics - "strings" — on the Coulomb branch which can meet in 3string junctions or end at the vacuum or singularities on the Coulomb branch. For example, in the $U(n) N=4$ SYM theory these "string" webs are thus stretched in the $6 n$-dimensional moduli space and end on $6(n-1)$-dimensional singular submanifolds. Note that this is different from the string web picture found in the string construction of this theory, where the strings are stretched in the 6-dimensional space transverse to $N$ D3-branes. It can be shown 1 that the 6 -dimensional string theory webs are obtained from our $6 n$-dimensional webs by a simple mapping. The basic reason this works is that the $6 N$-dimensional moduli space $\mathcal{M}$ of the $U(n)$ theory is $\mathcal{M}=\left(R^{6}\right)^{n} / S_{n}$ where the permutation group $S_{n}$ interchanging the $R^{6}$ factors is the Weyl group of $U(n)$.

Another property of the string webs found in string constructions is that 3pronged string states that stretch between D-branes satisfy charge conservation and tension balance. These properties are also reproduced 1 in the webs from our low energy solutions. However, the low energy solutions have an extended spatial structure which is not seen in the string constructions. For example, the configuration discussed above corresponds to a $(1,0)$ (fundamental string), a $(0,1)$ (D-string)and a $(1,1)$ string stretched between three D3-branes. The three prongs or string legs meet at a common point, the junction, which remains point-like even arbitrarily close to the CMS. In the field theory picture discussed here, we have seen that the leg corresponding to the decaying $(1,1)$ dyon grows in spatial size $(i . e$., along the D3-brane worldvolume) near the CMS. Arbitrarily close to the CMS, the configuration resembles two separate strings that end on the D3-brane corresponding to the decaying $U(1)$. Thus there does not appear to be a point-like junction as the decaying configuration approaches the CMS in the field theory picture.

The difference between the two perspectives is the result of different orders of limits. Consider the Dirac-Born-Infeld action for the D3-brane corresponding to the decaying dyon, treating it as a probe in a background of other D3-branes. The brane prong corresponding to the decaying $(1,1)$ dyon then ends on other D3-branes that are treated as a fixed background. Looking at the quadratic terms and comparing with the low energy effective action we have used above, we can see that the scalars $X$ in the field theory with mass dimension unity and the coordinates in the brane transverse space, $x$, are related by $X=x / \alpha^{\prime}$, where $\alpha^{\prime}$ is the string length squared. The separation between the charge centers in the D-brane worldvolume is

$$
r_{E B} \sim 1 /\left(-X_{0}\right)=\alpha^{\prime} /\left(-x_{0}\right) .
$$

The low energy field theory is a good approximation in the $\alpha^{\prime} \rightarrow 0$ limit holding the scalar vevs, including $X_{0}$, fixed. On the other hand, in the string junction/geodesic picture in string theory, the coordinates $x_{0}$ are what are held fixed: taking $\alpha^{\prime} \rightarrow 0$ to suppress higher stringy corrections gives a vanishing separation $r_{E B}$ between the charge centers, i.e. a pointlike junction. For $x_{0}=0$, the separation is indeterminate, which corresponds to the two strings ending anywhere on the D-brane. This recovers 
Decay of Supersymmetric Particles

the string theory result and it further illustrates that a spatially point-like junction is not visible within the field theory approximation.

\section{Acknowledgments}

It is a pleasure to thank A. Buchel, J. Hein, R. Maimon, J. Maldacena, M. Moriconi, S. Pelland, M. Rangamani, V. Sahakian, and A. Shapere for helpful comments and discussions. This work was supported in part by NSF grant PHY95-13717.

\section{References}

1. E. Witten and D. Olive, Phys. Lett. 78B (1978) 97.

2. N. Seiberg and E. Witten, Nucl. Phys. B426 (1994) 19; Nucl. Phys. B431 (1994) 484.

3. S. Cecotti and C. Vafa, Commun. Math. Phys. 158 (1993) 569.

4. P. Argyres and K. Narayan, hep-th/0101114.

5. A. Sen, Nucl. Phys. B475 (1996) 562; T. Banks, M. Douglas and N. Seiberg, Phys. Lett. B387 (1996) 278.

6. J. Gauntlett, C. Koehl, D. Mateos, P. Townsend and M. Zamaklar, Phys. Rev. D60 (1999) 045004.

7. K. Lee and P. Yi, Phys. Rev. D58 (1998) 066005; D. Bak, C. Lee, K. Lee and P. Yi, Phys. Rev. D61 (2000) 025001; D. Bak, K. Lee and P. Yi, Phys. Rev. D61 (2000) 045003; J. Gauntlett, N. Kim, J. Park and P. Yi, Phys. Rev. D61 (2000) 125012.

8. A. Ritz, M. Shifman, A. Vainshtein and M. Voloshin, hep-th/0006028 